\title{
A comparative study of air quality assessment before and during lockdown among Metro Cities of India
}

\author{
Chaturvedi P. ${ }^{1}$, Rathore K. S. ${ }^{2}$, Chaturvedi M. ${ }^{3}$ and Singh S. ${ }^{1}$ 冈
}

Received: 15.10 .2020

Revised: 12.11.2020

Accepted: 24.11.2020

\begin{abstract}
The aim of present study was to compare the air quality before and during lockdown due to COVID-19 pandemic at selected metro cities of India (Delhi, Kolkata, Bangalore, and Mumbai). The data of the selected parameters (Particulate Matter having diameter equal to or less than 2.5micron $\left(\mathrm{PM}_{2.5}\right)$, Particulate Matter having diameter equal to or less than 10micron $\left(\mathrm{PM}_{10}\right)$, Nitrogen oxides $\left(\mathrm{NO}_{2}\right)$, Ammonia $\left(\mathrm{NH}_{3}\right)$, Sulphur oxides $\left(\mathrm{SO}_{2}\right)$ Carbon monoxides $(\mathrm{CO})$, and $\mathrm{Ozone}\left(\mathrm{O}_{3}\right)$ for the present study was collected from the official website of Central pollution Control Board (CPCB) and analyzed by calculating mean, standard deviation, total variance, and correlation coefficient. Dendrogram analysis was also performed site wise. The concentration of all the parameters except ozone was found highest at Delhi among all the sites. Ozone values were found highest at Bangalore. A strong correlation was observed between $P M_{10}$ and $P M_{2.5}$ at all the sites during the study period. A great change in the values of all the studied parameters was observed before and during the lockdown periods. In metro cities values of $\mathbf{P M}_{2.5}$ was found higher than $\mathbf{P M}_{10}$ except at Bangalore where values of $\mathbf{P M} \mathbf{M}_{2.5}$ was found lower than $\mathrm{PM}_{10}$. Among all the studied metro cities, Delhi was found highly polluted before and during the lockdown period while Bangalore was found least polluted.
\end{abstract}

Key Words: Air Pollution, Covid-19, CPCB, Lockdown, Pollutant

\section{Introduction}

First lockdown during COVID-19 pandemic was declared by Government of India on March $22^{\text {nd }}$ 2020 and was continued in three regular phases up to $31^{\text {st }}$ May 2020. In India the first case of COVID19 was observed in Kerala on $30^{\text {th }}$ January 2020 and number increased to three by $3^{\text {rd }}$ February 2020; all passengers came from Wuhan, China, but there is no further rise in cases during the month of February 2020. On $4^{\text {th }}$ March 2020, 22 new patients came into highlight. These people were the members of a tourist group return back from Italy. The government of India followed lockdowns in whole country on $24^{\text {th }}$ March 2020 for 21 days and continued further for 3 more phases of 19,14 and 14 days respectively which ends on $31^{\text {st }}$ May 2020 . During the $3^{\text {rd }}$ and $4^{\text {th }}$ phase of lockdown some relaxations was given by Government of India, so that the financial activities were started with the opening of market and highways to maintain the Author's Address

${ }^{1}$ Department of Environmental Sciences, School of Sciences, ITM University, Gwalior (M.P.) India

${ }^{2}$ Department of Biotechnology, K. R. G. (Auto) P. G. College, Gwalior (M.P.) India

${ }^{3}$ School of Agriculture, ITM University, Gwalior (M.P.) India

E-mail.: shivomsingh101@gmail.com supply of food and daily needs and to maintain the economy of country and livelihood of the citizens. With the huge crowd of population of around 1.3 billion Indian has paid their responsibility towards the COVID-19. The WHO (World health organisation) and most of the countries continuously appreciates the strategy of Indian Government during this pandemic situation to break the chain of corona virus and to stop community transmission. During the initial phase of lockdown period, transportation and out-going of the peoples were strictly prohibited. The immediate effect of this action, downfall in air pollutant gases was noticed. Such gases directly affect the human health. It decreases the total lung capacity by chocking the alveoli of lungs, various gases are carcinogenic and toxic in nature. A study on SARS showed positive response of pollutant on long term exposure to pollution based on 2002/03 (Cui et al., 2003). These gases also disrupt agricultural practices, wild life and whole of the ecosystem directly hampered due to these pollutants. One of every eight death is directly related to the cause of air pollution. This situation is more dangerous in metro cities of India due to high level of air 
pollution especially in New Delhi, Mumbai, Bengaluru and Kolkata. Present study provides us a clear picture of air quality index of abovementioned cities under lockdown period and help strategy planners for future betterment.

\section{Material and Methods}

In present set of investigation, we analyzed data of initial 2020 up to lockdown period which is analyzed from National Air Quality Index (NAQI) of Central Pollution Control Board (CPCB) from $1^{\text {st }}$ January to $31^{\text {st }}$ May 2020. Data includes first two months of normal pollution trend of studied cities and the $3^{\text {rd }}$ month that is March under partial lockdown whereas April follows the strict implementation of lockdown. From May 2020 the opening of lockdown was considered and all the conditions were regularly monitored during this period. Data were collected from the official website https://app.cpcbccr.com/AQI_India of Central pollution Control Board (CPCB) and analyzed. All the data presenting here is the mean of a complete month with standard error value. Statistical analysis is done with the help of Minitab 18 software.

\section{Results and Discussion}

The results of all the studied sites (Delhi, Bangalore, Kolkata, and Mumbai) of all the studied parameters $\left(\mathrm{PM}_{10}, \mathrm{PM}_{2.5}, \mathrm{NO}_{2}, \mathrm{SO}_{2}, \mathrm{NH}_{3}, \mathrm{O}_{3}\right.$ and $\mathrm{CO})$ were given in table 1 to $10 \mathrm{~d}$ and figure $1-8 \mathrm{~d}$.

During and after lockdown period it was observed that the concentration of all the pollutants were decreased, only New Delhi and Mumbai showed increased concentration of $\mathrm{SO}_{2}$. The significant decrease in concentration of $\mathrm{PM}_{2.5}$ was observed during lockdown period i.e., March-June over January and February (Table1-4). Similar trends were observed in $\mathrm{PM}_{10}, \mathrm{NO}_{2}, \mathrm{NH}_{3}$ and $\mathrm{CO}$. The concentration of $\mathrm{O}_{3}$ and $\mathrm{SO}_{2}$ increased during lockdown phase because of powerplant and coal burning was allowed during this period. The $\mathrm{OH}$ radicle removed due to increase in atmospheric temperature and sunlight. Above mention line is responsible for high concentration of $\mathrm{O}_{3}$. This reaction is speeded up in warmer conditions and with more UV-light. Major photochemical paths for $\mathrm{O}_{3}$ sink are photolysis followed by the reaction of $\mathrm{O}$
(1D) with water vapor and via its reaction with hydrogen oxide radicals (HOx) (Monks, 2005;

Wayne, 2000).Central Pollution Control Board (CPCB) of India reported that Air Quality assessment improved during lockdown period and the concentration of pollutant gases decreased up to a certain level. Similar to Mumbai, In New Delhi AQI improved during lockdown period. The Mumbai city was less pollutant in compared to the New Delhi city because of three side sea surrounding and movement of air towards the sea breeze. The comparatively similar association between $\mathrm{SO}_{2}, \mathrm{NOx}$, and $\mathrm{CO}$ and meteorological parameters (temperature and relative humidity) was also found at another urban site (Kumari and Toshniwal, 2020). In figure-1 the trend of concentration of $\mathrm{PM}_{2.5}$ was found in order of, New Delhi $>$ Kolkata $>$ Mumbai $>$ Bangalore. The maximum concentration of $\mathrm{PM}_{2.5}$ recorded in New Delhi in month of January due to burning of agriculture waste. The emission of these pollutants increases the overall toxic burden of the environment, as well as deteriorate the ambient air quality (Ibe et al., 2017: Njoku and Ibe, 2009). Similarly decreasing trend was seen in the month of February and March and minimum in April and May. In Figure-2 the concentration of $\mathrm{PM}_{10}$ was observed as, New Delhi $>$ Kolkata $>$ Mumbai $>$ Bangalore. The elevated mean concentrations of $\mathrm{PM}_{10}$ across the locations could be attributed to the tendency of $\mathrm{PM}_{10}$ to be transported over short and long distances as a result of its particle size (Prospero et al., 1981). During the lockdown $\mathrm{PM}_{10}$ concentration decreases in New Delhi over other months due to decrease in the construction of roads, industrial work and other activity in NCR region. Figure-3 indicates different trend in concentration of $\mathrm{NO}_{2}$, the obseved concentration was in order Kolkata $>$ New Delhi $>$ Mumbai $>$ Bangalore. Sources of $\mathrm{NO}_{2}$ are wood burning in rural areas and fuel burning by automobiles. The lifetime of $\mathrm{NO}_{2}$ can vary with the photochemical environment, but is typically on the order of hours to a day (Seinfeld and Pandis, 1998) which further oxidizes into $\mathrm{HNO}_{3}$ and peroxyacetyl nitrate (PAN) (Sun et al., 2011). Highest concentration was observed in New Delhi and Kolkata during January and February due to heavy automobile load and industrial activity, however, gradually decline was found during lockdown period due to decrease in mentioned activities. 
A comparative study of air quality assessment

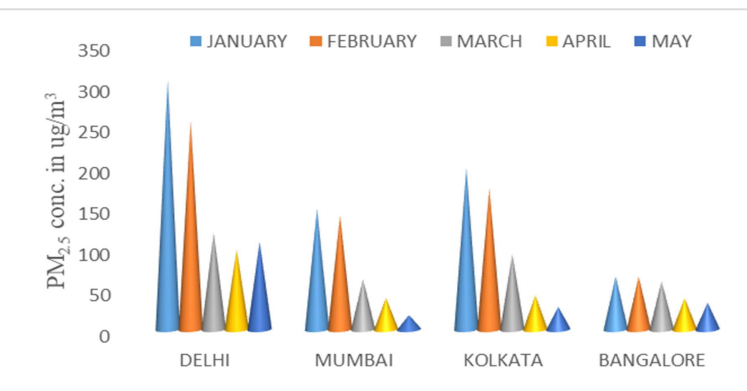

Figure 1. PM ${ }_{2.5}$ concentration at the different metro cities

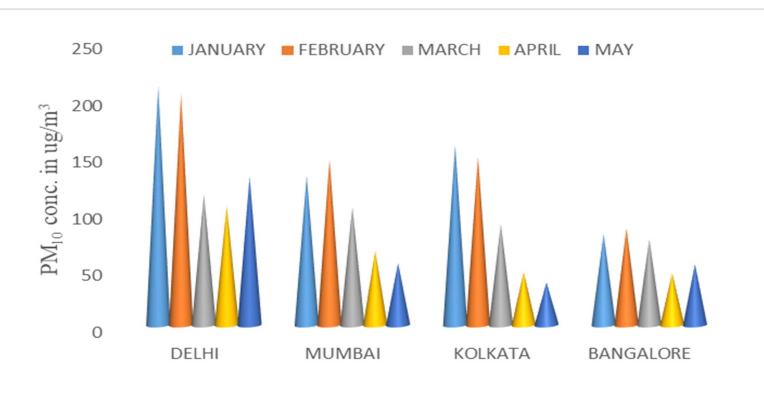

Figure 2. $\mathrm{PM}_{10}$ concentration at the different metro cities

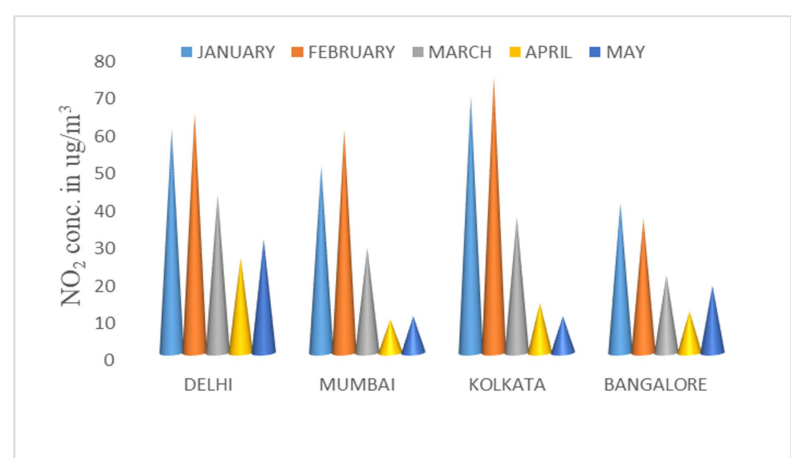

Figure 3. $\mathrm{NO}_{2}$ concentration at the different metro cities

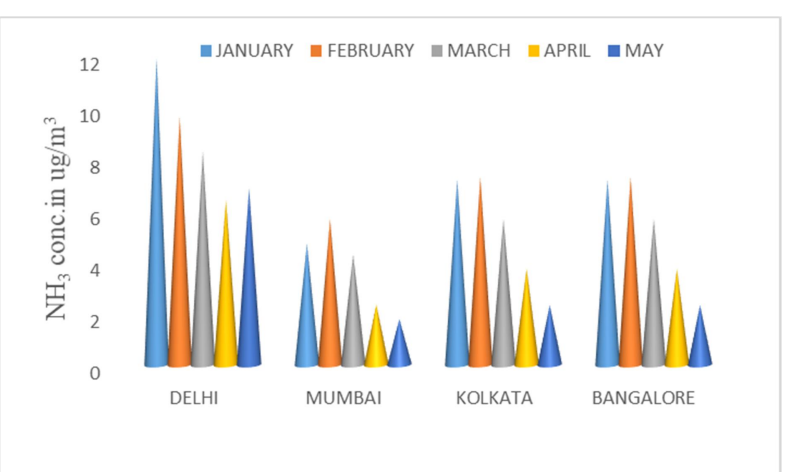

Figure 4. $\mathrm{NH}_{3}$ concentration at the different metro cities

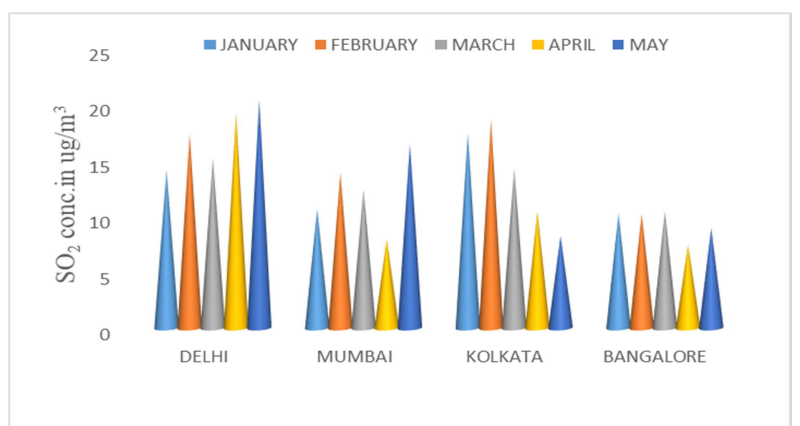

Figure 5. $\mathrm{SO}_{2}$ concentration at the different metro cities

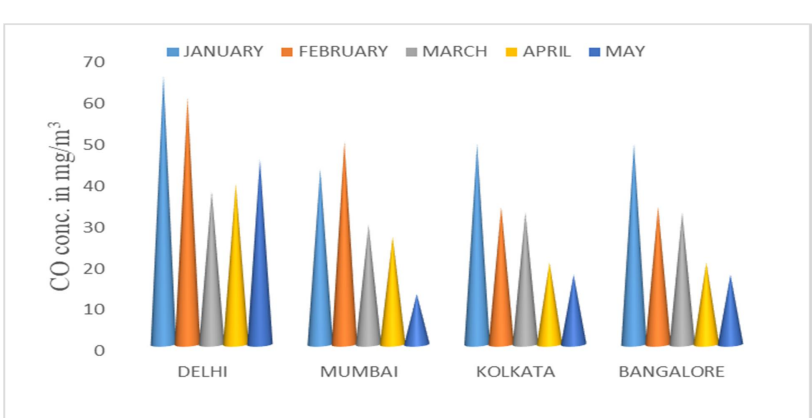

Figure 6. CO concentration at the different metro cities

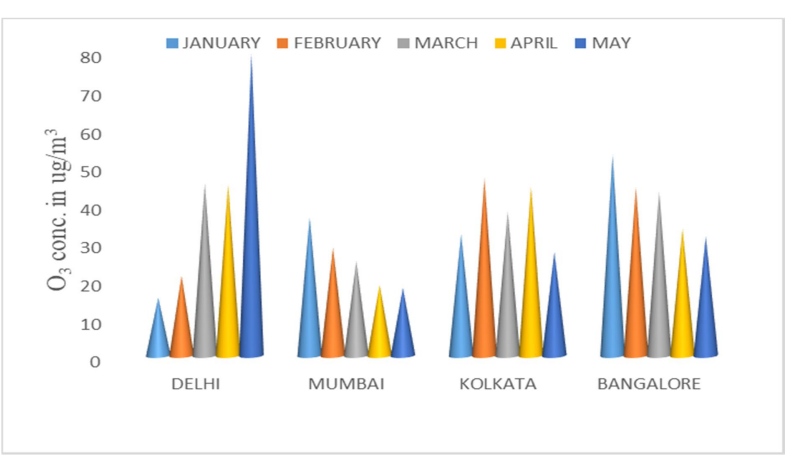

Figure 7. $\mathrm{O}_{3}$ concentration at the different metro cities

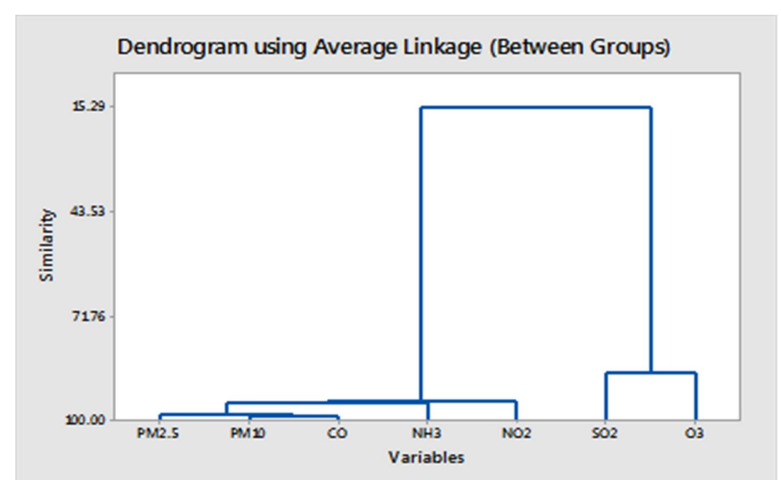

Figure 8a. The hierarchal cluster analysis of New Delhi city 


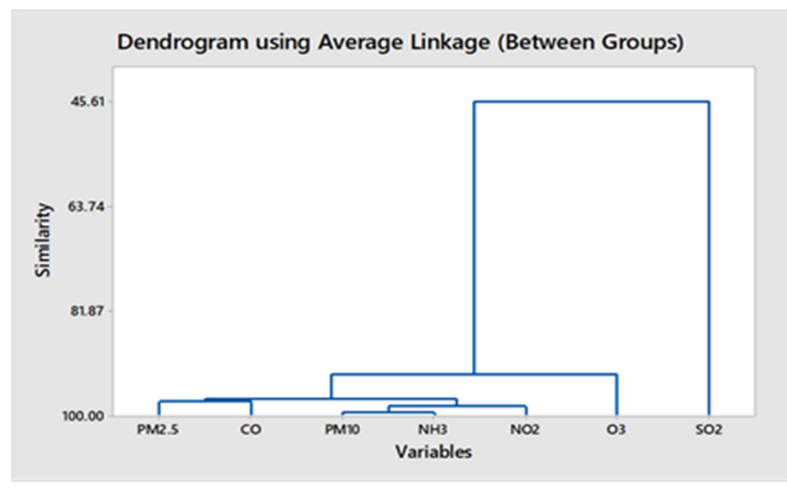

Figure 8b. The hierarchal cluster analysis of Mumbai city

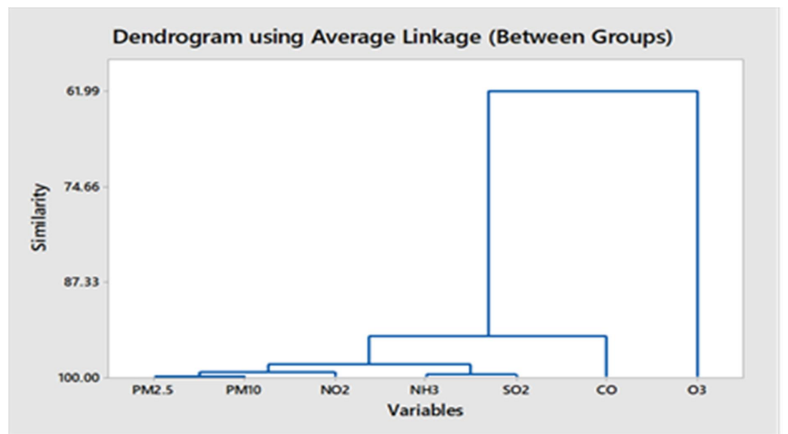

Figure 8c. The hierarchal cluster analysis of Kolkata city

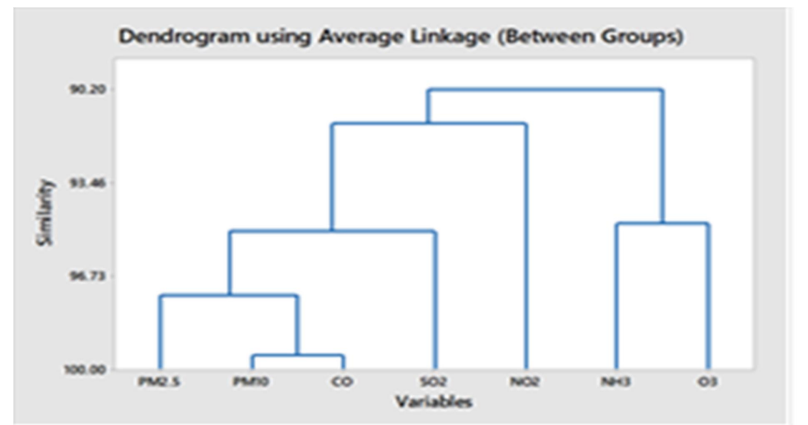

Figure 8d. The hierarchal cluster analysis of Bangalore

Furthermore, figure-4 shows maximum concentration of $\mathrm{NH}_{3}$ is New Delhi and minimum in Mumbai. $\mathrm{NH}_{3}$ has a residence time in the atmosphere of only a day or less, it contributes to the formation of atmosphere aerosols that reside in the atmosphere for several days to a week (Galperin and Sofiey, 1998).

The concentration of $\mathrm{SO}_{2}$ showed different trend at different places (figure-5). In India, about $60 \%$ of $\mathrm{SO}_{2}$ emissions may be due to the consumption of coal and oil products, mainly from industry (36\%) and transport (7.8\%), and others include biomass and non-energy consumption (Garg et al., 2001). Figure-6 indicates the trends of the $\mathrm{CO}$ concentration in order New Delhi $>$ Mumbai $>$ Kolkata $>$ Bangalore. The maximum concentration of CO was in New Delhi because of the major sources of $\mathrm{CO}$ are emission from fossil- and biofuel combustion, biomass burning, and oxidation of methane $\left(\mathrm{CH}_{4}\right)$ and non-methane hydrocarbon (NMHC) (Logan et al., 1981; Crutzen and Andreae 1990). Decrease in concentration was observed during the lockdown period due to decrease in above mentioned activities. The trends of pollutant gas $\mathrm{O}_{3}$ was found maximum in Bangalore and minimum in Mumbai. The high solar radiation intensity (i.e., temperature) has a direct influence on chemical kinetics and rates and the mechanism pathways for the $\mathrm{O}_{3}$ production (Pudasainee et al., 2006; Han et al., 2011). Other major factor in New Delhi is landlock condition by nearby NCR region. However, this study suggested that the negative $\mathrm{O}_{3}$ relative humidity correlation attributed to several other meteorological factors directly influencing $\mathrm{O}_{3}$ production (Tawfik and Steiner, 2013).

Figure-8a to figure-8d indicated the hierarchical cluster analysis (HCA) and was carried out to show the similarities and dissimilarities between the source and occurrence of the atmospheric pollutants. The result obtain from cluster analysis are represented as dendrogram. In figure $8 \mathrm{a}$ pollutants of New Delhi region $\left(\mathrm{PM}_{2.5}, \mathrm{PM}_{10}, \mathrm{NO}_{2}\right.$, $\mathrm{NH}_{3}, \mathrm{SO}_{2}$ show high similarity in cluster 1 where as it does not have any association with $\mathrm{CO}$ and $\mathrm{O}_{3}$. In Fig. 8b Mumbai city dendrogram shows cluster 1 as $\mathrm{SO}_{2}$ and does not have any similarity with other pollutants. In addition, in cluster $2 \mathrm{PM}_{2.5}, \mathrm{PM}_{10}$, $\mathrm{CO}, \mathrm{NH}_{3}, \mathrm{NO}_{2}$ and $\mathrm{O}_{3}$ depicts similarity with each other in a tree. In Figure 8c Kolkata dendrogram shows cluster 1 of $\mathrm{O}_{3}$ and does not have any association with other pollutants, whereas, rest all the pollutants in cluster 2 shows similarity with each other. Figure-8d depicts dendrogram of Bangalore city where all the pollutants in cluster 1 and 2 not form any association with each other hence all the pollutant show dissimilarity with each other. Table 5 and 6 are the total variance of the air pollutants and rotated component matrix of the atmospheric pollutants. Table 5 indicates that there are two components with initial Eigen value more than 1.00 . The result revealed that PC1 \& PC2 
A comparative study of air quality assessment

Table 1. Concentration of various pollutants in Delhi city before and during lockdown period

\begin{tabular}{|c|c|c|c|c|c|c|c|}
\hline \multirow[t]{2}{*}{ Months } & \multicolumn{7}{|c|}{ Air Quality Index Parameters } \\
\hline & $\begin{array}{c}\mathbf{P M}_{2.5} \\
\left(\mu \mathrm{g} / \mathrm{m}^{3}\right)\end{array}$ & $\begin{array}{c}\mathbf{P M}_{10} \\
\left(\mu \mathrm{g} / \mathbf{m}^{3}\right)\end{array}$ & $\frac{\mathrm{NO}_{2}}{\left(\mu \mathrm{g} / \mathrm{m}^{3}\right)}$ & $\begin{array}{c}\mathrm{NH}_{3} \\
\left(\mu \mathrm{g} / \mathrm{m}^{3}\right)\end{array}$ & $\begin{array}{c}\mathrm{SO}_{2} \\
\left(\mu \mathrm{g} / \mathrm{m}^{3}\right)\end{array}$ & $\begin{array}{c}\mathrm{CO} \\
\left(\mathrm{mg} / \mathrm{m}^{3}\right)\end{array}$ & $O_{3}\left(\mu \mathrm{g} / \mathrm{m}^{3}\right)$ \\
\hline January & $\begin{array}{l}305.300^{\mathrm{a}} \\
\pm 14.315\end{array}$ & $\begin{array}{l}210.373^{\mathrm{a}} \\
\pm 11.103\end{array}$ & $\begin{array}{c}59.576^{\mathrm{ab}} \\
\pm 3.056\end{array}$ & $\begin{array}{l}11.900^{\mathrm{a}} \\
\pm 0.377\end{array}$ & $\begin{array}{l}14.066^{\mathrm{c}} \\
\pm 0.419\end{array}$ & $\begin{array}{l}65.146^{\mathrm{a}} \\
\pm 4.191\end{array}$ & $\begin{array}{l}15.200^{\mathrm{b}} \\
\pm 1.086\end{array}$ \\
\hline February & $\begin{array}{l}254.446^{\mathrm{a}} \\
\pm 29.478\end{array}$ & $\begin{array}{l}203.500^{\mathrm{a}} \\
\pm 22.866\end{array}$ & $\begin{array}{l}63.940^{\mathrm{a}} \\
\pm 5.852\end{array}$ & $\begin{array}{l}9.630^{\mathrm{ab}} \\
\pm 0.351\end{array}$ & $\begin{array}{c}17.290^{\mathrm{abc}} \\
\pm 2.217\end{array}$ & $\begin{array}{c}59.666^{\mathrm{ab}} \\
\pm 7.070\end{array}$ & $\begin{array}{l}20.953^{b} \\
\pm 2.930\end{array}$ \\
\hline March & $\begin{array}{l}118.610^{b} \\
\pm 13.434\end{array}$ & $\begin{array}{l}115.526^{b} \\
\pm 16.129\end{array}$ & $\begin{array}{c}42.176^{\mathrm{bc}} \\
\pm 6.074\end{array}$ & $\begin{array}{c}8.300^{b} \\
\pm 0.799\end{array}$ & $\begin{array}{c}15.130^{\mathrm{bc}} \\
\pm 1.265\end{array}$ & $\begin{array}{l}37.053^{\mathrm{c}} \\
\pm 1.699\end{array}$ & $\begin{array}{l}45.160^{\mathrm{ab}} \\
\pm 10.014\end{array}$ \\
\hline April & $\begin{array}{c}107.103^{b} \\
\pm 3.545\end{array}$ & $\begin{array}{l}104.950^{b} \\
\pm 11.281\end{array}$ & $\begin{array}{l}25.313^{\mathrm{c}} \\
\pm 1.024\end{array}$ & $\begin{array}{c}6.413^{\mathrm{b}} \\
\pm 1.000\end{array}$ & $\begin{array}{c}19.183^{\mathrm{ab}} \\
\pm 1.529\end{array}$ & $\begin{array}{l}38.890^{\mathrm{c}} \\
\pm 0.735\end{array}$ & $\begin{array}{c}44.856^{\mathrm{ab}} \\
\pm 4.101\end{array}$ \\
\hline May & $\begin{array}{l}97.593^{b} \\
\pm 6.628\end{array}$ & $\begin{array}{l}107.14^{\mathrm{b}} \\
\pm 6.962\end{array}$ & $\begin{array}{l}30.323^{\mathrm{c}} \\
\pm 4.455\end{array}$ & $\begin{array}{c}6.846^{\mathrm{b}} \\
\pm 0.290\end{array}$ & $\begin{array}{l}20.373^{\mathrm{a}} \\
\pm 2.095\end{array}$ & $\begin{array}{c}44.753^{\mathrm{bc}} \\
\pm 1.938\end{array}$ & $\begin{array}{c}79.113^{\mathrm{a}} \\
\pm 17.414\end{array}$ \\
\hline
\end{tabular}

Table 2. Concentration of various pollutants in Mumbai city before and during lockdown period

\begin{tabular}{|c|c|c|c|c|c|c|c|}
\hline \multirow[t]{2}{*}{ Months } & \multicolumn{7}{|c|}{ Air Quality Index Parameters } \\
\hline & $\begin{array}{c}\mathbf{P M}_{2.5} \\
\left(\mu \mathrm{g} / \mathbf{m}^{3}\right)\end{array}$ & $\begin{array}{c}\mathrm{PM}_{10} \\
\left(\mu \mathrm{g} / \mathrm{m}^{3}\right)\end{array}$ & $\begin{array}{c}\mathrm{NO}_{2} \\
\left(\mu \mathrm{g} / \mathrm{m}^{3}\right)\end{array}$ & $\begin{array}{c}\mathrm{NH}_{3} \\
\left(\mu \mathrm{g} / \mathrm{m}^{3}\right)\end{array}$ & $\underset{\left(\mu \mathrm{SO} / \mathbf{m}^{3}\right)}{\mathrm{SO}}$ & $\begin{array}{c}\mathrm{CO} \\
\left(\mathrm{mg} / \mathrm{m}^{3}\right)\end{array}$ & $O_{3}\left(\mu \mathrm{g} / \mathrm{m}^{3}\right)$ \\
\hline January & $\begin{array}{l}147.670^{\mathrm{a}} \\
\pm 15.254\end{array}$ & $\begin{array}{c}131.700^{\mathrm{a}} \\
\pm 2.981\end{array}$ & $\begin{array}{c}49.786^{\mathrm{ab}} \\
\pm 0.477\end{array}$ & $\begin{array}{l}4.700^{\mathrm{a}} \\
\pm 0.310\end{array}$ & $\begin{array}{l}10.620^{\mathrm{a}} \\
\pm 0.909\end{array}$ & $\begin{array}{c}42.386^{\mathrm{ab}} \\
\pm 3.755\end{array}$ & $\begin{array}{l}36.313^{\mathrm{a}} \\
\pm 6.444\end{array}$ \\
\hline February & $\begin{array}{c}139.693^{\mathrm{a}} \\
\pm 9.816\end{array}$ & $\begin{array}{c}146.053^{\mathrm{a}} \\
\pm 7.395\end{array}$ & $\begin{array}{l}59.370^{\mathrm{a}} \\
\pm 4.373\end{array}$ & $\begin{array}{l}5.666^{\mathrm{a}} \\
\pm 0.235\end{array}$ & $\begin{array}{l}13.850^{\mathrm{a}} \\
\pm 2.457\end{array}$ & $\begin{array}{l}49.046^{\mathrm{a}} \\
\pm 3.558\end{array}$ & $\begin{array}{l}28.323^{\mathrm{a}} \\
\pm 3.437\end{array}$ \\
\hline March & $\begin{array}{l}61.450^{b} \\
\pm 8.910\end{array}$ & $\begin{array}{c}104.150^{\mathrm{ab}} \\
\pm 17.821\end{array}$ & $\begin{array}{c}28.013^{\mathrm{bc}} \\
\pm 8.901\end{array}$ & $\begin{array}{l}4.290^{\mathrm{a}} \\
\pm 0.395\end{array}$ & $\begin{array}{l}12.340^{\mathrm{a}} \\
\pm 1.712\end{array}$ & $\begin{array}{c}28.963^{b c} \\
\pm 4.561\end{array}$ & $\begin{array}{l}24.670^{\mathrm{a}} \\
\pm 2.784\end{array}$ \\
\hline April & $\begin{array}{l}38.440^{b} \\
\pm 4.935\end{array}$ & $\begin{array}{c}65.336^{b c} \\
\pm 7.269\end{array}$ & $\begin{array}{l}8.926^{\mathrm{c}} \\
\pm 1.036\end{array}$ & $\begin{array}{l}2.343^{b} \\
\pm 0.130\end{array}$ & $\begin{array}{c}7.943^{\mathrm{a}} \\
\pm 1.269\end{array}$ & $\begin{array}{c}26.050^{\mathrm{bc}} \\
\pm 6.777\end{array}$ & $\begin{array}{l}18.520^{\mathrm{a}} \\
\pm 1.893\end{array}$ \\
\hline May & $\begin{array}{l}17.696^{\mathrm{b}} \\
\pm 2.228\end{array}$ & $\begin{array}{l}54.676^{\mathrm{c}} \\
\pm 7.629\end{array}$ & $\begin{array}{l}9.796^{\mathrm{c}} \\
\pm 1.243 \\
\end{array}$ & $\begin{array}{c}1.773^{b} \\
\pm 0.259\end{array}$ & $\begin{array}{l}16.426^{\mathrm{a}} \\
\pm 4.707\end{array}$ & $\begin{array}{l}12.203^{\mathrm{c}} \\
\pm 0.579\end{array}$ & $\begin{array}{l}17.796^{\mathrm{a}} \\
\pm 3.299\end{array}$ \\
\hline
\end{tabular}

Table 3. Concentration of various pollutants in Kolkata City before and during lockdown period

\begin{tabular}{|c|c|c|c|c|c|c|c|}
\hline \multirow{2}{*}{ Months } & \multicolumn{7}{|c|}{ Air Quality Index Parameters } \\
\cline { 2 - 8 } & $\mathbf{P M}_{\mathbf{2 . 5}}$ & $\begin{array}{c}\mathbf{P M}_{\mathbf{1 0}} \\
\left(\boldsymbol{\mu g} / \mathbf{m}^{\mathbf{3}}\right)\end{array}$ & $\begin{array}{c}\mathbf{N O}_{\mathbf{2}} \\
\left(\boldsymbol{\mu g} / \mathbf{m}^{\mathbf{3}}\right)\end{array}$ & $\begin{array}{c}\mathbf{N H}_{\mathbf{3}} \\
\left(\boldsymbol{\mu g} / \mathbf{m}^{\mathbf{3}}\right)\end{array}$ & $\begin{array}{c}\mathbf{S O}_{\mathbf{2}} \\
\left(\boldsymbol{\mu g} / \mathbf{m}^{\mathbf{3}}\right)\end{array}$ & $\begin{array}{c}\mathbf{C O} \\
\left(\mathbf{m g} / \mathbf{m}^{\mathbf{3}}\right)\end{array}$ & $\mathbf{O}_{\mathbf{3}}\left(\boldsymbol{\mu g} / \mathbf{m}^{\mathbf{3}}\right)$ \\
\hline January & $197.853^{\mathrm{a}}$ & $158.613^{\mathrm{a}}$ & $68.413^{\mathrm{a}}$ & $7.196^{\mathrm{a}}$ & $17.390^{\mathrm{a}}$ & $48.520^{\mathrm{a}}$ & $31.890^{\mathrm{a}}$ \\
& \pm 8.701 & \pm 7.823 & \pm 4.857 & \pm 0.413 & \pm 0.947 & \pm 3.829 & \pm 7.661 \\
\hline February & $172.173^{\mathrm{a}}$ & $148.040^{\mathrm{a}}$ & $73.530^{\mathrm{a}}$ & $7.306^{\mathrm{a}}$ & $18.566^{\mathrm{a}}$ & $33.296^{\mathrm{b}}$ & $46.480^{\mathrm{a}}$ \\
& \pm 10.375 & \pm 7.729 & \pm 4.584 & \pm 0.443 & \pm 1.346 & \pm 2.066 & \pm 5.171 \\
\hline March & $92.333^{\mathrm{b}}$ & $89.036^{\mathrm{b}}$ & $36.320^{\mathrm{b}}$ & $5.680^{\mathrm{a}}$ & $14.210^{\mathrm{ab}}$ & $31.930^{\mathrm{b}}$ & $37.683^{\mathrm{a}}$ \\
& \pm 13.305 & \pm 12.298 & \pm 7.839 & \pm 0.416 & \pm 1.150 & \pm 3.677 & \pm 8.808 \\
\hline April & $41.896^{\mathrm{c}}$ & $46.763 \mathrm{c}$ & $13.263^{\mathrm{c}}$ & $3.733^{\mathrm{b}}$ & $10.396^{\mathrm{bc}}$ & $19.826^{\mathrm{c}}$ & $44.270^{\mathrm{a}}$ \\
& \pm 13.017 & \pm 11.263 & \pm 1.960 & \pm 0.400 & \pm 1.276 & \pm 1.954 & \pm 6.172 \\
\hline May & $27.706^{\mathrm{c}}$ & $37.726^{\mathrm{c}}$ & $9.813^{\mathrm{c}}$ & $2.310^{\mathrm{b}}$ & $8.256^{\mathrm{c}}$ & $16.906^{\mathrm{c}}$ & $27.173^{\mathrm{a}}$ \\
& \pm 1.236 & \pm 2.293 & \pm 1.706 & \pm 0.489 & \pm 1.095 & \pm 0.978 & \pm 2.031 \\
\hline
\end{tabular}


Chaturvedi et al.

Table 4. Concentration of various pollutants in Bengalore City before and during lockdown period

\begin{tabular}{|c|c|c|c|c|c|c|c|}
\hline \multirow[t]{2}{*}{ Months } & \multicolumn{7}{|c|}{ Air Quality Index Parameters } \\
\hline & $\begin{array}{c}\mathbf{P M}_{2.5} \\
\left(\mu \mathrm{g} / \mathrm{m}^{3}\right)\end{array}$ & $\begin{array}{c}\mathrm{PM}_{10} \\
\left(\mu \mathrm{g} / \mathrm{m}^{3}\right)\end{array}$ & $\begin{array}{c}\mathrm{NO}_{2} \\
\left(\mu \mathrm{g} / \mathrm{m}^{3}\right)\end{array}$ & $\begin{array}{c}\mathrm{NH}_{3} \\
\left(\mu \mathrm{g} / \mathrm{m}^{3}\right)\end{array}$ & $\begin{array}{c}\mathrm{SO}_{2} \\
\left(\mu \mathrm{g} / \mathrm{m}^{3}\right)\end{array}$ & $\begin{array}{c}\mathrm{CO} \\
\left(\mathrm{mg} / \mathrm{m}^{3}\right)\end{array}$ & $O_{3}\left(\mu \mathrm{g} / \mathrm{m}^{3}\right)$ \\
\hline January & $\begin{array}{l}65.000^{\mathrm{a}} \\
\pm 4.380\end{array}$ & $\begin{array}{l}80.226^{\mathrm{ab}} \\
\pm 4.833\end{array}$ & $\begin{array}{l}39.943^{\mathrm{a}} \\
\pm 1.828\end{array}$ & $\begin{array}{l}3.393^{\mathrm{a}} \\
\pm 0.232\end{array}$ & $\begin{array}{l}10.226^{\mathrm{a}} \\
\pm 0.549\end{array}$ & $\begin{array}{l}48.446^{\mathrm{a}} \\
\pm 2.305\end{array}$ & $\begin{array}{l}52.663^{\mathrm{a}} \\
\pm 6.914\end{array}$ \\
\hline February & $\begin{array}{l}64.853^{\mathrm{a}} \\
\pm 3.110\end{array}$ & $\begin{array}{l}85.326^{\mathrm{a}} \\
\pm 1.558\end{array}$ & $\begin{array}{c}35.730^{\mathrm{ab}} \\
\pm 8.124\end{array}$ & $\begin{array}{l}3.203^{\mathrm{a}} \\
\pm 0.151\end{array}$ & $\begin{array}{l}10.173^{\mathrm{a}} \\
\pm 1.668\end{array}$ & $\begin{array}{l}48.756^{\mathrm{a}} \\
\pm 2.191\end{array}$ & $\begin{array}{c}44.103^{\mathrm{ab}} \\
\pm 3.517\end{array}$ \\
\hline March & $\begin{array}{l}59.080^{\mathrm{ab}} \\
\pm 10.262\end{array}$ & $\begin{array}{l}75.706^{\mathrm{ab}} \\
\pm 10.356\end{array}$ & $\begin{array}{c}20.793^{\mathrm{abc}} \\
\pm 4.689\end{array}$ & $\begin{array}{l}3.080^{\mathrm{a}} \\
\pm 0.147\end{array}$ & $\begin{array}{l}10.433^{\mathrm{a}} \\
\pm 0.334\end{array}$ & $\begin{array}{l}47.190^{\mathrm{a}} \\
\pm 0.776\end{array}$ & $\begin{array}{c}43.013^{\mathrm{ab}} \\
\pm 0.916\end{array}$ \\
\hline April & $\begin{array}{c}38.496^{\mathrm{ab}} \\
\pm 4.812\end{array}$ & $\begin{array}{l}45.580^{c} \\
\pm 3.520\end{array}$ & $\begin{array}{c}17.976^{\mathrm{bc}} \\
\pm 0.335\end{array}$ & $\begin{array}{l}2.776^{\mathrm{a}} \\
\pm 0.205\end{array}$ & $\begin{array}{l}7.423^{\mathrm{a}} \\
\pm 0.556\end{array}$ & $\begin{array}{l}29.343^{b} \\
\pm 2.399\end{array}$ & $\begin{array}{l}33.220^{b} \\
\pm 1.172\end{array}$ \\
\hline May & $\begin{array}{l}32.853^{b} \\
\pm 3.974\end{array}$ & $\begin{array}{l}53.710^{\mathrm{bc}} \\
\pm 2.522\end{array}$ & $\begin{array}{l}11.106^{\mathrm{c}} \\
\pm 1.073\end{array}$ & $\begin{array}{l}2.106^{b} \\
\pm 0.212\end{array}$ & $\begin{array}{l}8.926^{\mathrm{a}} \\
\pm 0.296\end{array}$ & $\begin{array}{l}34.116^{b} \\
\pm 2.283\end{array}$ & $\begin{array}{l}31.22^{b} \\
\pm 2.665\end{array}$ \\
\hline
\end{tabular}

Values are represented as mean $\pm \mathrm{SE}$

*Levels not connected by the same letters in horizontal row are monthly significantly different.

Table 5. Total variance of air pollutants concentration for Delhi city

\begin{tabular}{|c|c|c|c|c|c|c|c|c|c|}
\hline \multirow{2}{*}{$\begin{array}{l}\text { Co } \\
\text { mpo } \\
\text { nent }\end{array}$} & \multicolumn{3}{|c|}{ Initial Eigenvalues } & \multicolumn{3}{|c|}{$\begin{array}{l}\text { Extraction Sums of Squared } \\
\text { Loadings }\end{array}$} & \multicolumn{3}{|c|}{$\begin{array}{l}\text { Rotation Sums of Squared } \\
\text { Loadings }\end{array}$} \\
\hline & Total & $\begin{array}{l}\% \text { of } \\
\text { variances }\end{array}$ & Cumulative & Total & $\begin{array}{l}\% \text { of } \\
\text { variances }\end{array}$ & Cumulative & Total & $\begin{array}{l}\% \text { of } \\
\text { variances }\end{array}$ & $\begin{array}{l}\text { Cumulativ } \\
\text { e }\end{array}$ \\
\hline 1 & 5.185 & 74.100 & 74.100 & 5.185 & 74.100 & 74.100 & 4.015 & 57.400 & 57.400 \\
\hline 2 & 1.195 & 17.100 & 91.100 & 1.195 & 17.100 & 91.100 & 1.410 & 20.100 & 77.500 \\
\hline 3 & 0.279 & 4.000 & 95.100 & & & & & & \\
\hline 4 & 0.168 & 2.400 & 97.500 & & & & & & \\
\hline 5 & 0.115 & 1.600 & 99.200 & & & & & & \\
\hline 6 & 0.044 & 0.600 & 99.800 & & & & & & \\
\hline 7 & 0.014 & 0.200 & 100.000 & & & & & & \\
\hline
\end{tabular}

Table 6. Total variance of air pollutants concentration for Mumbai city

\begin{tabular}{|c|c|c|c|c|c|c|c|c|c|}
\hline \multirow[t]{2}{*}{ Component } & \multicolumn{3}{|c|}{ Initial Eigenvalues } & \multicolumn{3}{|c|}{$\begin{array}{l}\text { Extraction Sums of Squared } \\
\text { Loadings }\end{array}$} & \multicolumn{3}{|c|}{$\begin{array}{l}\text { Rotation Sums of Squared } \\
\text { Loadings }\end{array}$} \\
\hline & Total & $\begin{array}{l}\% \text { of } \\
\text { variances }\end{array}$ & Cumulative & & Total & $\begin{array}{l}\% \text { of } \\
\text { variances }\end{array}$ & Cumulative & & Total \\
\hline 1 & 5.028 & 71.800 & 71.800 & 5.028 & 71.800 & 71.800 & 4.319 & 61.700 & 61.700 \\
\hline 2 & 1.119 & 16.000 & 87.800 & 1.119 & 16.000 & 87.800 & 1.288 & 18.400 & 80.100 \\
\hline 3 & 0.556 & 7.900 & 95.800 & & & & & & \\
\hline 4 & 0.181 & 2.600 & 98.300 & & & & & & \\
\hline 5 & 0.063 & 0.900 & 99.300 & & & & & & \\
\hline 6 & 0.034 & 0.500 & 99.700 & & & & & & \\
\hline 7 & 0.018 & 0.300 & 100.000 & & & & & & \\
\hline
\end{tabular}

Table 7. Total variance of air pollutants concentration for Kolkata city

\begin{tabular}{|l|l|l|l|l|l|l|l|l|l|}
\hline \multirow{2}{*}{ Component } & \multicolumn{3}{|l|}{ Initial Eigenvalues } & \multicolumn{2}{l|}{$\begin{array}{l}\text { Extraction Sums of Squared } \\
\text { Loadings }\end{array}$} & \multicolumn{3}{l|}{$\begin{array}{l}\text { Rotation Sums of Squared } \\
\text { Loadings }\end{array}$} \\
\cline { 2 - 11 } & Total & $\begin{array}{l}\text { \% of } \\
\text { variances }\end{array}$ & Cumulative & & Total & $\begin{array}{l}\text { \% of } \\
\text { variances }\end{array}$ & Cumulative & & Total \\
\hline 1 & 5.622 & 80.300 & 80.300 & 5.622 & 80.300 & 80.300 & 5.372 & 76.700 & 76.700 \\
\hline 2 & 1.052 & 15.000 & 95.300 & 1.052 & 15.000 & 95.300 & 1.088 & 15.600 & 92.300 \\
\hline 3 & 0.131 & 1.900 & 97.200 & & & & & & \\
\hline 4 & 0.091 & 1.300 & 98.500 & & & & & & \\
\hline 5 & 0.087 & 1.200 & 99.700 & & & & & & \\
\hline 6 & 0.014 & 0.200 & 99.900 & & & & & & \\
\hline 7 & 0.004 & 0.001 & 100.000 & & & & & & \\
\hline
\end{tabular}

182

Environment Conservation Journal 
A comparative study of air quality assessment

Table 8. Total variance of air pollutants concentration for Banglore city

\begin{tabular}{|l|l|l|l|l|l|l|l|l|l|}
\hline \multirow{2}{*}{ Component } & \multicolumn{3}{|l|}{ Initial Eigenvalues } & \multicolumn{2}{l|}{$\begin{array}{l}\text { Extraction Sums of Squared } \\
\text { Loadings }\end{array}$} & \multicolumn{3}{l|}{ Rotation Sums of Squared Loadings } \\
\cline { 2 - 11 } & Total & $\begin{array}{l}\text { \% of } \\
\text { variances }\end{array}$ & Cumulative & & Total & $\begin{array}{l}\text { \% of } \\
\text { variances }\end{array}$ & Cumulative & & Total \\
\hline 1 & 5.062 & 72.300 & 72.300 & 5.062 & 72.300 & 72.300 & 1.366 & 19.500 & 19.500 \\
\hline 2 & 0.803 & 11.500 & 83.800 & 0.803 & 11.500 & 83.800 & 1.344 & 19.200 & 38.700 \\
\hline 3 & 0.447 & 6.400 & 90.200 & & & & & & \\
\hline 4 & 0.258 & 3.700 & 93.900 & & & & & & \\
\hline 5 & 0.233 & 3.300 & 97.200 & & & & & & \\
\hline 6 & 0.202 & 2.600 & 99.800 & & & & & & \\
\hline 7 & 0.012 & 0.200 & 100.000 & & & & & & \\
\hline
\end{tabular}

Table 9. Rotated Component matrix

\begin{tabular}{|c|c|c|c|c|c|c|c|c|}
\hline \multirow{2}{*}{ Variable } & \multicolumn{2}{|c|}{ Delhi } & \multicolumn{2}{c|}{ Mumbai } & \multicolumn{2}{c|}{ Kolkata } & \multicolumn{2}{c|}{ Banglore } \\
\cline { 2 - 9 } & $\mathbf{1}$ & $\mathbf{2}$ & $\mathbf{1}$ & $\mathbf{2}$ & $\mathbf{1}$ & $\mathbf{2}$ & $\mathbf{1}$ & $\mathbf{2}$ \\
\hline $\mathrm{PM} 2.5$ & 0.919 & -0.191 & 0.890 & 0.389 & 0.988 & -0.053 & 0.481 & 0.312 \\
\hline $\mathrm{PM} \mathrm{10}$ & 0.953 & -0.005 & 0.924 & 0.297 & 0.990 & -0.062 & 0.269 & 0.362 \\
\hline $\mathrm{NO}_{2}$ & 0.828 & -0.179 & 0.923 & 0.299 & 0.989 & -0.092 & 0.263 & 0.310 \\
\hline $\mathrm{NH}_{3}$ & 0.718 & -0.448 & 0.876 & 0.266 & 0.918 & -0.129 & 0.878 & 0.160 \\
\hline $\mathrm{SO}_{2}$ & -0.070 & 0.968 & 0.030 & 0.092 & 0.901 & -0.217 & 0.151 & 0.927 \\
\hline $\mathrm{CO}$ & 0.953 & -0.113 & 0.967 & 0.128 & 0.882 & -0.124 & 0.383 & 0.366 \\
\hline $\mathrm{O}_{3}$ & -0.385 & 0.438 & 0.344 & 0.930 & 0.070 & -0.997 & 0.481 & 0.312 \\
\hline
\end{tabular}

Table 10a. Correlation analysis of atmospheric pollutants of New Delhi city

\begin{tabular}{|l|l|l|l|l|l|l|l|}
\hline Parameters & $\mathbf{P M}_{2.5}$ & $\mathbf{P M}_{\mathbf{1 0}}$ & $\mathbf{N O}_{\mathbf{2}}$ & $\mathbf{N H}_{\mathbf{3}}$ & $\mathbf{S O}_{2}$ & $\mathbf{C O}$ & $\mathbf{O}_{\mathbf{3}}$ \\
\hline $\mathbf{P M}_{2.5}$ & 1 & & & & & & \\
\hline $\mathbf{P M}_{\mathbf{1 0}}$ & 0.975 & 1 & & & & & \\
\hline $\mathbf{N O}_{2}$ & 0.921 & 0.920 & 1 & & & & \\
\hline $\mathbf{N H}_{\mathbf{3}}$ & 0.951 & 0.886 & 0.896 & 1 & & & \\
\hline $\mathbf{S O}_{\mathbf{2}}$ & -0.640 & -0.495 & -0.679 & -0.826 & 1 & & \\
\hline $\mathbf{C O}$ & 0.966 & 0.985 & 0.845 & 0.864 & -0.436 & 1 & \\
\hline $\mathbf{O}_{3}$ & -0.830 & -0.725 & -0.808 & -0.809 & 0.742 & -0.690 & 1 \\
\hline
\end{tabular}

Table 10b. Correlation analysis of atmospheric pollutants of Mumbai city

\begin{tabular}{|l|l|l|l|l|l|l|l|}
\hline Parameters & $\mathbf{P M}_{\mathbf{2 . 5}}$ & $\mathbf{P M}_{\mathbf{1 0}}$ & $\mathbf{N O}_{\mathbf{2}}$ & $\mathbf{N H}_{\mathbf{3}}$ & $\mathbf{S O}_{\mathbf{2}}$ & $\mathbf{C O}$ & $\mathbf{O}_{\mathbf{3}}$ \\
\hline $\mathbf{P M}_{2.5}$ & 1 & & & & & & \\
\hline $\mathbf{P M}_{\mathbf{1 0}}$ & 0.956 & 1 & & & & & \\
\hline $\mathbf{N O}_{\mathbf{2}}$ & 0.965 & 0.985 & 1 & & & & \\
\hline $\mathbf{N H}_{\mathbf{3}}$ & 0.899 & 0.987 & 0.954 & 1 & & & \\
\hline $\mathbf{S O}_{\mathbf{2}}$ & -0.127 & -0.033 & 0.072 & -0.029 & 1 & & \\
\hline $\mathbf{C O}$ & 0.950 & 0.954 & 0.935 & 0.932 & -0.252 & 1 & \\
\hline $\mathbf{O}_{3}$ & 0.931 & 0.869 & 0.862 & 0.805 & -0.155 & 0.808 & 1 \\
\hline
\end{tabular}

Table 10c. Correlation analysis of atmospheric pollutants of Kolkata city

\begin{tabular}{|l|l|l|l|l|l|l|l|}
\hline Parameters & $\mathbf{P M}_{\mathbf{2 . 5}}$ & $\mathbf{P M}_{\mathbf{1 0}}$ & $\mathbf{N O}_{\mathbf{2}}$ & $\mathbf{N H}_{\mathbf{3}}$ & $\mathbf{S O}_{\mathbf{2}}$ & $\mathbf{C O}$ & $\mathbf{O}_{\mathbf{3}}$ \\
\hline $\mathbf{P M}_{\mathbf{2 . 5}}$ & 1 & & & & & & \\
\hline $\mathbf{P M}_{\mathbf{1 0}}$ & 0.950 & 1 & & & & & \\
\hline $\mathbf{N O}_{\mathbf{2}}$ & 0.835 & 0.876 & 1 & & & & \\
\hline $\mathbf{N H}_{\mathbf{3}}$ & 0.926 & 0.764 & 0.696 & 1 & & & \\
\hline $\mathbf{S O}_{\mathbf{2}}$ & 0.820 & 0.930 & 0.755 & 0.567 & 1 & & \\
\hline $\mathbf{C O}$ & 0.945 & 0.990 & 0.844 & 0.761 & 0.960 & 1 & \\
\hline $\mathbf{O}_{3}$ & 0.932 & 0.862 & 0.878 & 0.897 & 0.767 & 0.879 & 1 \\
\hline
\end{tabular}


Chaturvedi et al.

Table 10d. Correlation analysis of atmospheric pollutants of Bangalore city

\begin{tabular}{|l|l|l|l|l|l|l|l|}
\hline Parameters & $\mathbf{P M}_{\mathbf{2 . 5}}$ & $\mathbf{P M}_{\mathbf{1 0}}$ & $\mathbf{N O}_{\mathbf{2}}$ & $\mathbf{N H}_{\mathbf{3}}$ & $\mathbf{S O}_{\mathbf{2}}$ & $\mathbf{C O}$ & $\mathbf{O}_{\mathbf{3}}$ \\
\hline $\mathbf{P M}_{\mathbf{2 . 5}}$ & 1 & & & & & & \\
\hline $\mathbf{P M}_{\mathbf{1 0}}$ & 0.998 & 1 & & & & & \\
\hline $\mathbf{N O}_{\mathbf{2}}$ & 0.983 & 0.991 & 1 & & & & \\
\hline $\mathbf{N H}_{\mathbf{3}}$ & 0.953 & 0.964 & 0.963 & 1 & & & \\
\hline $\mathbf{S O}_{\mathbf{2}}$ & 0.959 & 0.972 & 0.982 & 0.993 & 1 & & \\
\hline $\mathbf{C O}$ & 0.931 & 0.918 & 0.862 & 0.886 & 0.856 & 1 & \\
\hline $\mathbf{O}_{\mathbf{3}}$ & 0.181 & 0.210 & 0.279 & 0.389 & 0.395 & -0.018 & 1 \\
\hline
\end{tabular}

accounts for $57.400 \%$ and $20.100 \%$ of the variance respectively. Result obtained from table 6 suggested that while $\mathrm{PM}_{2.5}, \mathrm{PM}_{10}, \mathrm{NO}_{2}, \mathrm{NH}_{3}$, and $\mathrm{CO}$ have positive loading in PC1 with correlation coefficient value of $0.919,0.953,0.823$, and 0.718 respectively. Similarly, $\mathrm{SO}_{2}$ has a high loading in PC2 with correlation coefficient of 0.968 . It should be noted that all the pollutants are not correlated.

Table 11 and 12 are the total variance of the air pollutants and rotated component matrix of the atmospheric pollutants $\left(\mathrm{PM}_{2.5}, \mathrm{PM}_{10}, \mathrm{NO}_{2}, \mathrm{NH}_{3}\right.$, $\mathrm{SO}_{2}, \mathrm{CO}, \mathrm{O}_{3}$ ) at Bangalore city at the normal and lockdown phase. The table 11 indicates that there is one component with initial Eigen value greater than 1.0. The result revealed that $\mathrm{PC1}$ and $\mathrm{PC} 2$ accounted for $19.500 \%$ and $19.200 \%$ of the variance respectively. Result obtained from table suggested that $\mathrm{PM}_{2.5}, \mathrm{PM}_{10}, \mathrm{NO}_{2}, \mathrm{NH}_{3}, \mathrm{SO}_{2}, \mathrm{CO}$, and $\mathrm{O}_{3}$ have positive loading with values in $\mathrm{PC} 1$ and $\mathrm{PC} 2$ with correlation coefficient. Correlation analysis of New Delhi city during the normal and lockdown is presented in table 10a. The result revealed a strong positive correlation between $\mathrm{PM}_{2.5}$ and $\mathrm{PM}_{10}(\mathrm{r}=0.975), \mathrm{PM}_{2.5}$ and $\mathrm{NO}_{2}(\mathrm{r}=$ $0.921), \mathrm{PM}_{2.5}$ and $\mathrm{NH}_{3}(\mathrm{r}=0.951)$ and $\mathrm{PM}_{2.5}$ and $\mathrm{CO}$ $(\mathrm{r}=0.966)$. similarly, $\mathrm{PM}_{10}$ and $\mathrm{NO}_{2}(\mathrm{r}=0.920)$, $\mathrm{PM}_{10}$ and $\mathrm{NH}_{3}(\mathrm{r}=0.886), \mathrm{PM}_{10}$ and $\mathrm{CO}(\mathrm{r}=0.985)$, $\mathrm{NH}_{3}$ and $\mathrm{NO}_{2}(\mathrm{r}=0.896)$ a moderate positive correlation is observed between $\mathrm{O}_{3}$ and $\mathrm{SO}_{2}$, where as a negative correlation observed which are not statistically significant. Correlation analysis of the Mumbai city during the normal and lockdown phase is presented in the table $10 \mathrm{~b}$. The strong positive correlation is observed between $\mathrm{PM}_{2.5}$ and $\mathrm{PM}_{10}(\mathrm{r}=0.956), \mathrm{PM}_{2.5}$ and $\mathrm{NO}_{2}(\mathrm{r}=0.965), \mathrm{PM}_{2.5}$ and $\mathrm{NH}_{3}(\mathrm{r}=0.899), \mathrm{PM}_{2.5}$ and $\mathrm{CO}(\mathrm{r}=0.950)$, $\mathrm{PM}_{2.5}$ and $\mathrm{O}_{3}(\mathrm{r}=0.931)$. Similarly, most of the correlations are strongly positive but few relations are partially negative correlated. Correlation analysis of the Kolkata city during the normal and lockdown phase is presented in the table 10c. Mostly strong positive correlation was observed between the parameters but a week correlation was also seen between $\mathrm{PM}_{2.5}$ and $\mathrm{O}_{3}(\mathrm{r}=0.181), \mathrm{PM}_{10}$ and $\mathrm{O}_{3}(\mathrm{r}=0.210), \mathrm{NO}_{2}$ and $\mathrm{O}_{3}(\mathrm{r}=0.279) \mathrm{NH}_{3}$ and $\mathrm{O}_{3}(\mathrm{r}=0.389)$ and $\mathrm{SO}_{2}$ and $\mathrm{O}_{3}(\mathrm{r}=0.395)$. In addition, a very week correlation is observed between $\mathrm{CO}$ and $\mathrm{O}_{3}(\mathrm{r}=0.018)$. Correlation analysis of the Bangalore city during the normal and lockdown phase is presented in the table 10b. There is strong positive correlation between most of the pollutants. similarly, a moderate positive correlation is observed between $\mathrm{NH}_{3}$ and $\mathrm{NO}_{2}(\mathrm{r}$ $=0.696), \mathrm{NH}_{3}$ and $\mathrm{SO}_{2}(\mathrm{r}=0.567), \mathrm{NH}_{3}$ and $\mathrm{CO}(\mathrm{r}$ $=0.761), \mathrm{SO}_{2}$ and $\mathrm{O}_{3}(\mathrm{r}=0.767)$.

\section{Conclusion}

It should be eminent that scientific fact provides a clear picture in policy making for the future prospects and making the guideline for hence Lockdown phase of COVID-19 pandemic is a great boon to environment and wild life. While all the anthropogenic activities caused reduction in pollutant level of various gases measured by CPCB. AAQR provides a clear view of reduction in pollutant level in major cities viz., Mumbai, Kolkata, New Delhi and Bengaluru. Lockdown period provides a devastating fact about reduction in air pollution level in major cities and also provide an ideology for its cause and control efforts. The efforts should be nature friendly which include alternate source of energy to reduce the pollution level and technological measures for reduction in emission level so that air quality standard should be maintained for nature friendly future. The concentration of all the parameters except ozone was found highest at Delhi among all the sites. Order of the studied metro cities was found as Delhi $>$ Mumbai $>$ Kolkata $>$ Bangalore. 


\section{A comparative study of air quality assessment}

\section{Acknowledgements}

Authors are thankful to Central Pollution Control Board (CPCB), National Ambient Air Quality

\section{References}

Cui, Y., Zhang, Z. F., Froines, J., Zhao, J., Wang, H., Yu, S. Z. and Detels, R. 2003. Air pollution and case fatality of SARS in the People's Republic of China: an ecologic study. Environment Health Global, (2): 15. https://doi.org/10.1186/1476-069X-2-15.

Crutzen, P. J. and Andreae, M. O. 1990. Biomass burning in the tropics: impact on atmospheric chemistry and biogeochemical cycles. Science, 250(4988): 1669-1678. doi:10.1126/science.250.4988.1669.

Galperin, M. V. and Sofiev M. A. 1998. The long-range transport of ammonia and ammonium in the Northern Hemisphere. Atmospheric Environment, 32: 373-80. doi:10.1016/S1352-2310(97)00045-9.

Garg, A., Shukla, P. R., Bhattacharya, S. and Dadhwal, V. K. 2001. Sub-region (district) and sector level $\mathrm{SO}_{2}$ and $\mathrm{NOx}$ emissions for India: assessment of inventories and mitigation flexibility. Atmospheric Environment, 35(4): 703-713.

Han, S., Bian, H., Feng, Y., Liu, A., Li, X., Zeng, F. and Zhang, X. 2011. Analysis of the relationship between $\mathrm{O}_{3}$, $\mathrm{NO}$ and $\mathrm{NO}_{2}$ in Tianjin, China. Aerosol and Air Quality Research, (11): 128-139. doi:10.4209/aaqr.2010.07.0055.

Ibe, F. C., Opara, A. I., Njoku, P. C. and Alinnor, J. I. 2017. Ambient air quality assessment of Orlu, Southeastern Nigeria, Journal of Applied Science, 17: 441-451.

Kumari, P. and Toshniwal, D. 2020. Impact of lockdown measures during COVID-19 on air quality-A case study of India. International journal of Environmental Health Research,

https://doi.org/10.10 80/09603123.2020.1778646

Logan, J. A., Prather, M. J., Wofsy, S. C. and McElroy, M. B. 1981.Tropospheric chemistry: a global perspective.
Monitoring Program (NAAQMP) for providing daily data on website.

Journal of Geophysical Research, 86C8: 7210-7254. doi:10.1029/JC086iC08p07210

Monks, P. S. 2005. Gas-phase radical chemistry in the troposphere. Chemical Society Reviews, 34(5): 376-395.

Njoku, P. C. and Ibe, F. C. 2009. Heavy metal accumulation in electronic technicians, Journal of the chemical Society Nigeria, 34(1): 1-4.

Prospero, J. M., Glaccum, R. A. and Nees, R. T. 1981. Atmospheric transport of soil dust from Africa to South America. Nature, 28(9): 570-572.

Pudasainee, D., Sapkota, B., Shrestha, M. L., Kaga, A., Kondo, A. and Inoue, Y. 2006. Ground level ozone concentrations and its association with $\mathrm{NOx}$ and meteorological parameters in Kathmandu valley. Nepal Atmosphere Environment, 40(40): 8081-8087. doi: 10.1016/j.atmosenv.2006.07.011

Seinfeld, J. H. and Pandis, S. N. 1998. Atmospheric chemistry and physics. From Air Pollution to Climate Changes.Wiley, New York.

Sun, Y., Wang, L., Wang, Y., Quan, L. and Zirui, L. 2011. In situ measurements of $\mathrm{SO}_{2}, \mathrm{NOx}, \mathrm{NOy}$, and $\mathrm{O} 3$ in Beijing, China during august 2008. Science of Total Environment, 4095: 933-940.

Tawfik, A. B. and Steiner, A. L. 2013. A proposed physical mechanism for ozone-meteorology correlations using landatmosphere coupling regimes. Atmosphere Environment, 72: $50-59$.

Wayne, R. P. 2000. Chemistry of atmospheres: an introduction to the chemistry of the atmospheres of earth, the planets, and their satellites. Oxford University Press, New York. 